\title{
Mobile robot with failure inspection system for ferromagnetic structures using magnetic memory method
}

\author{
N. J. Montes de Oca-Mora ${ }^{1}$ - R. M. Woo-Garcia ${ }^{1}$ ' R. Juarez-Aguirre ${ }^{1}$ - A. L. Herrera-May ${ }^{1} \cdot$ A. Sanchez-Vidal ${ }^{2}$. \\ C. A. Ceron-Alvarez ${ }^{2} \cdot J_{\text {. Restrepo }}^{3} \cdot$ I. Algredo-Badillo ${ }^{4} \cdot$ F. Lopez-Huerta ${ }^{2}$ (D)
}

Received: 1 October 2020 / Accepted: 14 October 2021

Published online: 03 November 2021

(c) The Author(s) $2021 \quad$ OPEN

\begin{abstract}
Flaws or cracks are one of the major failures in oil and gas pipeline networks. The early detection of these failures is very important for the safety of the industry, and this last requires of analysis for non-destructive testing (NDT), which is reliable, inexpensive and easy to implement. In this paper, we propose the development of an embedded prototype mounted on a mobile robot for the inspection of defects in ferromagnetic plates. This prototype has two embedded systems (control and data acquisition), which are based on a microcontroller of 8 and 32 bits, respectively. On the one hand, the first system for control has the logic to govern the sensors and motors that will allow to the robot moves with autonomous way during $45 \mathrm{~min}$. While, the second system presents an algorithm for storing, processing and sending the data obtained from the sensors, being able to measure variations in the magnetic field in the order of $0.1 \mu$ T. Magneticfield reading tests have been carried out on control ASTM A-27 ferromagnetic plates, obtaining experimental response in the 3 axes of the magnetic domains, which is very close to the expected results by the magnetic-flux density model that is calculated from the fields $E$ and B derived from the equations of a Hertz dipole, and developed in the high-level Python programming language. The prototype proposed for NDT can detect geometric defects in the range of millimeters, producing changes in the density of the magnetic field in the order of thousands of $\mu$ T.
\end{abstract}

Keywords Magnetic field · Magnetic memory $\cdot$ Magnetic sensor $\cdot$ Non-destructive testing $\cdot$ Phyton

\section{Introduction}

Detecting damages in structures such as buildings, aircraft, vehicles or pipelines is one of the aspects that have been studied and analyzed in every branch of engineering to avoid breakdowns, malfunctions or deficiencies that could lead to risks of accidents or high unforeseen economic costs [1-3]. To prevent this, several approaches are necessary such as visual inspection with highly specialized operators, preventive maintenance, and exclusive techniques for damage detection. For this reason, at present, it is necessary to corroborate the integrity of both public and private structures to ensure their safety. Thus, different experimental techniques have been used to evaluate deformation in structures such as digital holographic interferometry to learn to what extent the beam is deformed at the load and how much it can withstand [4], o the experimental research about mechanical properties of

F. Lopez-Huerta, frlopez@uv.mx | ${ }^{1}$ Research Center in Micro and Nanotechnology, Universidad Veracruzana, Ruiz Cortines 455, Boca del Río, 94294 Xalapa, Ver, Mexico. ${ }^{2}$ Faculty of Electrical and Electronic Engineering, Universidad Veracruzana, Calzada Ruiz Cortines 455, Boca del Río, 94294 Xalapa, Ver, Mexico. ${ }^{3}$ Grupo de Magnetismo Y Simulación, Instituto de Física, Universidad de Antioquia, A.A. 1226, Medellín, Colombia. ${ }^{4}$ Computer Science Department, National Institute of Astrophysics, Optics and Electronics (INAOE), Luis Enrique Erro 1, Santa María Tonanzintla, 72840 Puebla, Mexico. 
the high-strength steel S960 QL and its welded joints, carried out in fatigue-testing equipment at the laboratory level [5]. An important example occurs in the oil industry, which makes use of pipelines for the supply and transport of hydrocarbons in a liquid or gaseous state, requiring that such pipelines must be in optimal conditions for their use, therefore it is necessary to analyze their structure to detect possible failures or leaks that could endanger both personnel and distribution.

It is important to highlight that the mentioned analysis requires an extensive inspection process and, in some cases, demands for material to be analyzed, which is submitted to certain tests that affect its proper functioning or even cause the total loss of it, this type of analysis is known as destructive testing. Since it is very costly and there are some inconveniences when performing structural analysis by the tests or destructive tests, alternative methods are required, which do not interfere with regular activities, nor affect or damage the element to be analyzed. These methods are known as non-destructive tests, such as the magnetic memory method, eddy currents, and active vision using light scattering.

The optical NDT methods provide higher sensitivity and resolution for high precision applications, however, requires a high cost of implementation and have better penetration on most of dry non-metallic or insolating materials [6].

The advantage of the Magnetic Memory Method (MMM) over other non-destructive testing methods that apply magnetism, such as Magnetic Particle Testing, is that MMM does not require the artificial magnetization of the element to be tested $[2,7]$, but rather makes use of its residual magnetic field for the detection of faults or anomalies. This occurs in structures of a ferromagnetic nature. Another advantage of this method is the capacity not only to detect the superficial anomalies but also the internal ones that the element or structure can have. Magnetoresistive sensors have the ability to detect magnetic field disturbances, which is of great importance for the detection of anomalies in ferromagnetic structures when using the MMM $[8,9]$. Such distortion may be a reaction of some defect in the structure, which can be flaws, fractures, hits or dents. The advantages of this method are mainly: (a) the detection of faults in the structure by means of a non-destructive analysis $[1,10]$, which is very economic and practical solution in the industry, (b)the possibility of detecting both external and internal faults in the structure, this feature is highly appreciated in the preventive maintenance of structures [11], and (c) there is an increasing production of sensors for the detection of magnetic fields due to the technological advances, and these sensors have multiple applications in different fields like monitoring and/or control of industrial processes [12], detection of hydrocarbon leaks [13], medicine, deformation of aeronautical structures, and petrochemical tanks [14]. For this reason, it is still necessary to develop new systems that meet the requirements of existing market needs, easy to use, low cost, efficient and portable.

Embedded systems are part of most of today's technological devices, which are usually composed of digital, analog, mixed-mode circuits and modules of one or several communication protocols. With the tendency to add up all the Systems on Chip (SoC), it is possible to find them in different applications, such as aerial controls, traffic signals, and robots. These lasts are very relevant, since they enable to reach hostile or dangerous places for the human being. The field of mobile robots is of great interest today and their use in structural failure analysis techniques is increasing [15]. Many of them are already used in non-destructive studies [16], and others are already employed in the field of ferromagnetic materials [17]. Even so, their use is still in development, so every day new types of mobile robots are created, and they are capable of performing different types of tasks $[18,19]$.

For example, a highly maneuverable mobile omniwheel robot is experimentally characterized in [20] for simple trajectories of motion using four $12 \mathrm{~V} \mathrm{DC}$ motors with PID controllers, the system proposed is only suitable for movement at different angles without changing the orientation of the platform and is expected to be applied in computer vision system to bypass obstacles and navigation systems with graphic interfaces. Currently, there are electronic instrumentation equipment that include graphic interfaces for their manipulation, this equipment is adjusted to the specific needs of each application context. Generally, the interfaces have a design that is friendly and intuitive for the end user, some of these have certain modules that allow the handling of data in a fast and simple way. Also, the equipment includes communication modules based on universal protocols that allow the interaction between a personal computer (PC) and the system in an efficient way. This equipment is designed to do specific tasks, so the end user must adapt to the established parameters and does not have the possibility of expanding or modifying the measurement capabilities [21]. To solve this drawback, one can choose to design and implement virtual instruments based on software, sensors, interface cards and PCs. This can provide important advantages, which include data storage capacity, integration of alternative communication protocols, new operating systems and software portability.

This study develops a land-based mobile prototype for the detection of defects in ferromagnetic structures using the magnetic memory method. The detection prototype is formed by two embedded systems: a) the first has the purpose of performing the translational displacement of 
Fig. 1 Block diagram system for the mobile robot

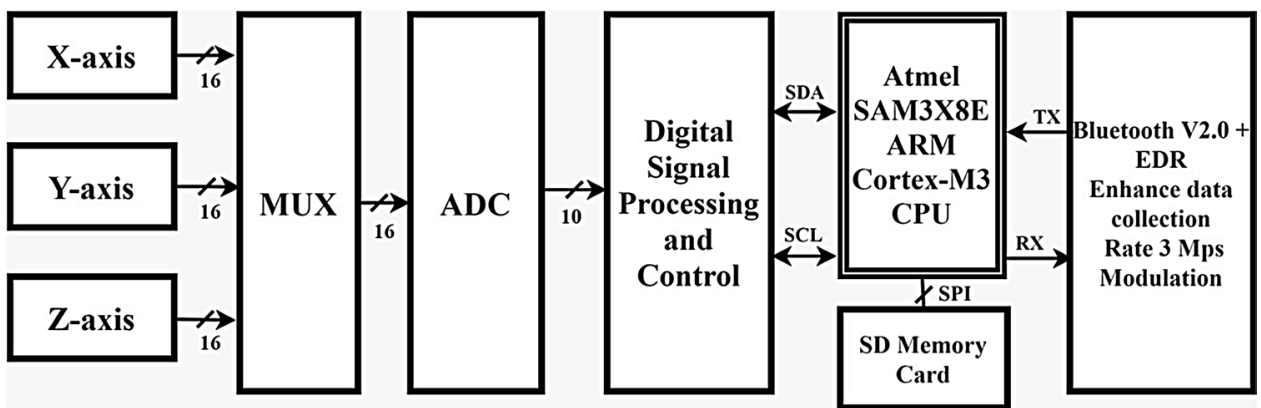

the robot on the earth surface through an object-oriented logic, it has the control module of the infrared sensors, motors and tracks, and b)the second consists of a magnetoresistive microsensor, a module for the data transmission via Bluetooth and a module for data processing. In addition, the latter maintains the communication, storage and processing of information obtained by the microsensor when performs the inspection.

This paper is organized as follows; in Sect. 2, we describe the mobile robot system composed of two blocks control and data acquisition, and the Python simulations of the magnetic field in rectangular defects. The measurement results of the ferromagnetic plate defects are described in Sect. 3. Next, in Sect. 4, we describe the discussions of the mobile robot system. Finally, we present the conclusions and future avenues of research in Sect. 5.

\section{Materials and methods}

\subsection{Design of the mobile robot and signal processing system}

The experimental method used to analyze ferromagnetic structures is based on reading, sampling and processing the residual magnetic field from the structure that is being tested. The alteration of the magnetic field is due to the presence of irregularities present in the structure. These irregularities, measured through the proposed system, are displayed in the graphical user interface (GUI) implemented for sampling, processing and storing the data obtained from the measurements. The data processing is generated in the embedded system, which has MAG3110 built-in commercial magnetic microsensors, capable of detecting magnetic field variations in a linear detection range of $\pm 1000 \mu \mathrm{T}$, with a sensitivity of $0.10 \mu \mathrm{T}$, this microsensor has an analog-digital converter, a filter and a multiplexer [22]. The embedded system is based on a 32-bit Atmel SAMD21 microcontroller, allowing a high digitalization of the data obtained from the scanning that the magnetic microsensor performs on the board. Then, the data are sent to a PC via the control protocol and adaptation of the logical link, using the HC-05 Bluetooth module. Also, the measurements can be store in a microSD, Fig. 1 shows the block diagram system. The complete system is within a structure designed and printed in 3D, which allows to the prototype to be linked with a mobile device for the analysis of structures of different sizes, this leads to the system in a reconfigurable device, as shown in Fig. 2.

The mobile robot can move in different topographic conditions and can be programmed to perform paths at different travel speeds $(2.15-8.6 \mathrm{~cm} / \mathrm{s})$. For the current study, the speed of $2.15 \mathrm{~cm} / \mathrm{s}$ was established to capture the variations of the magnetic field due to the defects present in a ferromagnetic plate as a test object. The ferromagnetic plate has dimensions of $76.5 \times 17 \mathrm{~cm}$ and a thickness of $6.7 \mathrm{~mm}$. Six cuts have been made along the plate in order to emulate rectangular defects, see Fig. 3 . The cuts are separated at a distance of $10 \mathrm{~cm}$ in an equidistant manner and are characterized exclusively by having the same length of the incision, as shown in Table 1. Three pairs of cuts were made to determine the reproducibility of the system. Figure 4 shows the arrangement designed to perform the detection of the variations of the magnetic field, the separation between the plate and the microsensor is

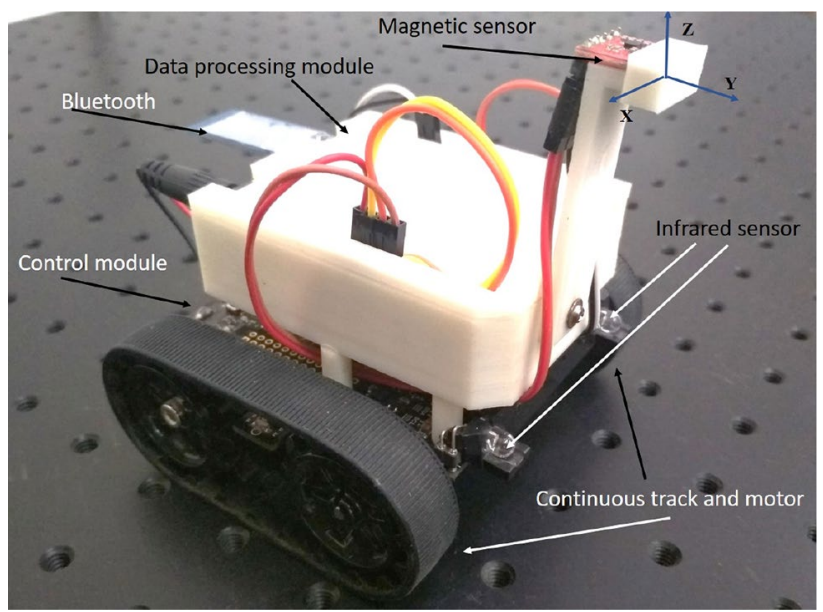

Fig. 2 Prototype executed for the inspection system of defects in ferromagnetic structures 




Fig. 3 Cuts made to the structure to emulate defects in the ferromagnetic plate

Table 1 Dimensions the cuts proposed in this study

\begin{tabular}{llll}
\hline Cuts & Width $(\mathrm{mm})$ & Large $(\mathrm{mm})$ & Depth $(\mathrm{mm})$ \\
\hline A1, A2 & 1 & 40 & 2.5 \\
B1, B2 & 3 & 40 & 3 \\
C1, C2 & 5 & 40 & 3 \\
\hline
\end{tabular}

$0.5 \mathrm{~cm}$, while the speed is kept constant along the axis of the plate.

A Finite State Machine (FSM) shows the robot's behavior path in five states, see Fig. 5. The FSM always starts in 'START' state, waiting for $100 \mathrm{~ms}$ time required for setting registers and assigning variables, after this, FSM changes to 'FOLLOW' state, activating both motors and following a straight line, if at some point the robot detects an obstacle in the right side, then it stops and waits for $100 \mathrm{~ms}$ stopping the engine before a spin change because the variations in engine revs are not immediately translated into changes in drive shaft rotation speed and, after, goes in to "LEFT TURN" (stopping left motor), until there is no obstacles on the right side. If some obstacle exists, then it will return to "FOLLOW" state, however, when it is in "LEFT TURN" state and detects presence of an object on the left side, regardless of whether or not there is an object on right side, the robot stops $100 \mathrm{~ms}$ and changes to "BACKWARD" state. On the other hand, if the robot is in "FOLLOW" state and senses an obstacle on the left side, it stops

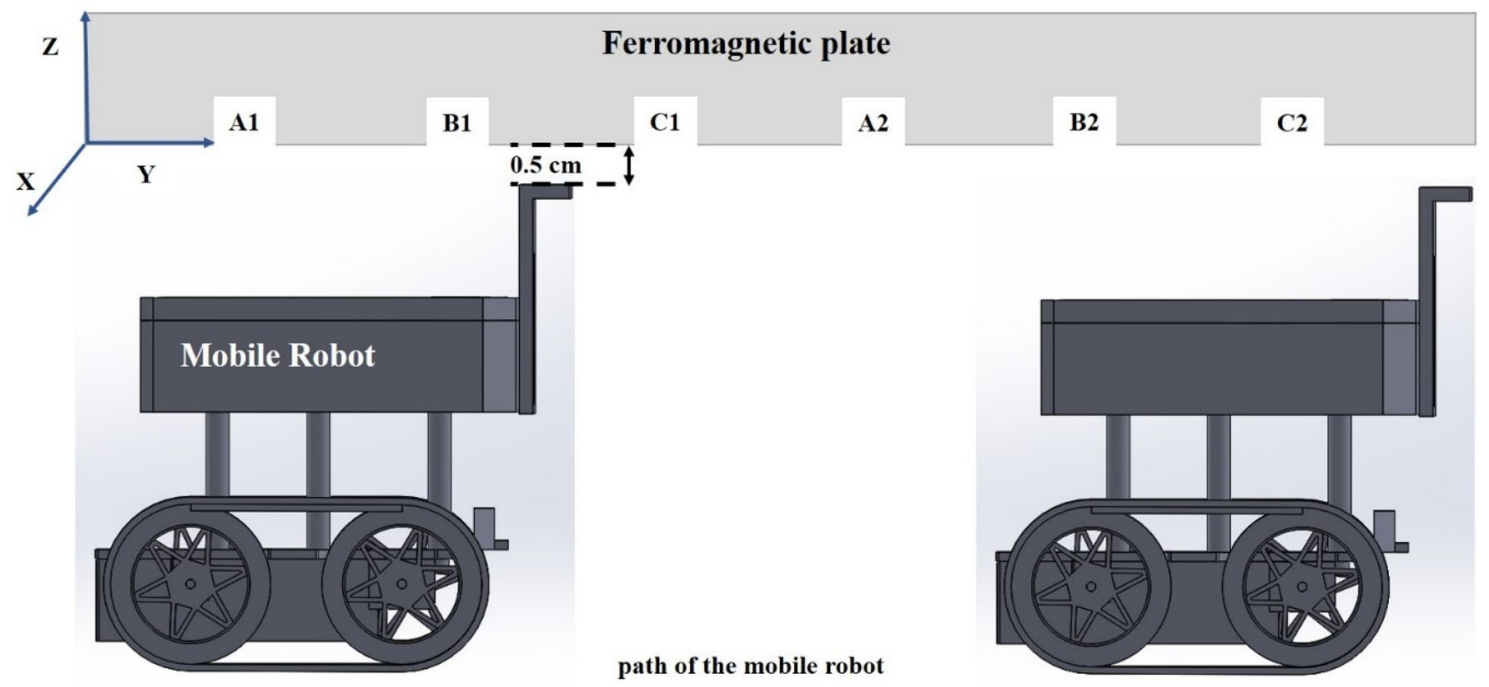

Fig. 4 Proposed arrangement for the path of the mobile robot along a ferromagnetic plate 


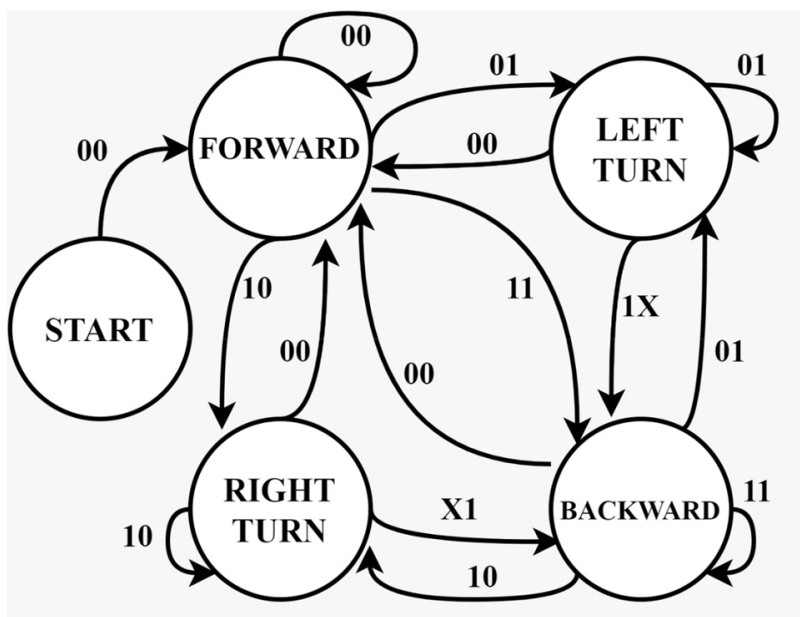

Inputs: Infrared sensors left \& right

Fig. 5 FSM of robot's behavior path

$100 \mathrm{~ms}$ and switches to "RIGHTTURN" state. If the right sensor detects an object, then it will go to "BACKWARD" state. Finally, if robot is in "FOLLOW" state and is full blocking its path, then it will change to "BACKWARD" state, being able to change from this state to "RIGHT TURN" if there is no obstacle on the left side or to "LEFT TURN" if not there is an obstacle on the right side.

\subsection{Python simulations of the magnetic field in rectangular defect}

In order to elucidate the relationship among the profiles of the magnetic field measured by the sensor and geometric characteristics of the flaws, numerical simulations were performed. In the simulations, the system was considered as formed by an ordered arrangement of magnetic moments representing the scenario of a magnetized system incorporating in the structure's rectangular flaws of different aspect ratios. Hence, the magnetic flux density (strength of the B-field) in Teslas was determined by adding the contributions to the magnetic field of every magnetic moment (representing an average domain) at a given distance from the surface. Every dipolar contribution, being the curl of the vector potential $A$, reads as follows

$\vec{B}(\vec{r})=\vec{\nabla} \times \vec{A}=\frac{\mu_{0}}{4 \pi}\left[\frac{3 \vec{r}(\vec{m} \cdot \vec{r})}{r^{5}}-\frac{\vec{m}}{r^{3}}\right]$

where $m$ is the magnetic moment per domain, and $r$ is the vector going from the position of every magnetic moment in the arrangement to the position where $B$ is evaluated. By repeating iteratively this procedure, the cartesian components of $B$ were obtained along the profile at a given distance from the surface. The corresponding experimental counterpart was set up using a pair of permanent neodymium magnets, as shown in Fig. 6 , in order to magnetize the domains in the region where the sensor came through. Figure 7 shows the array of random and ordered magnetic domains using Python. The Fig. 8, 9 and 10 shows the behavior of the magnetic field on the plate under test by using the model implemented in software and written in Python programming language and running in a workstation Dell T7910.

\section{Results}

In this section, measurements made through the MMM to a rectangular ASTM A-27 steel plate are presented, which has 6 defects that are mechanically induced. The complete system is powered by four $1.5 \mathrm{~V}$ batteries (see Fig. 11), which has an autonomy of $45 \mathrm{~min}$ and a power consumption of approximately $2 \mathrm{~W}$. Additionally, the microsensor has a resolution of $0.1 \mu \mathrm{T}$, and the information obtained from it can be stored in a microSD or sent
Fig. 6 Magnetization of the plate to orient the magnetics domain a top view, $\mathbf{b}$ side view of the setup using a permanent neodymium magnet


SN Applied Sciences A SPRINGER NATURE journal 
(a)

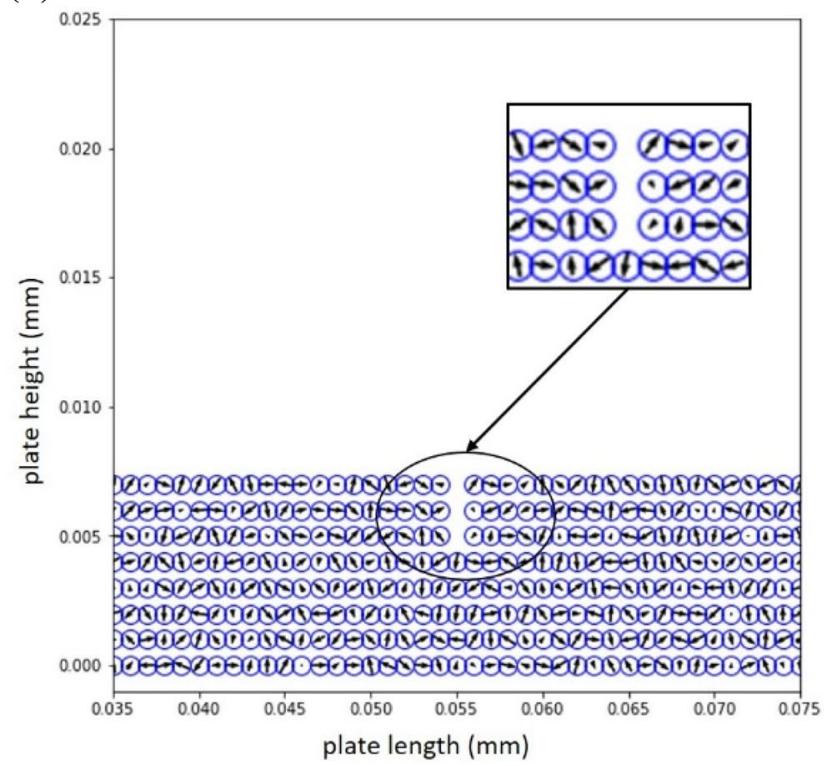

(b)

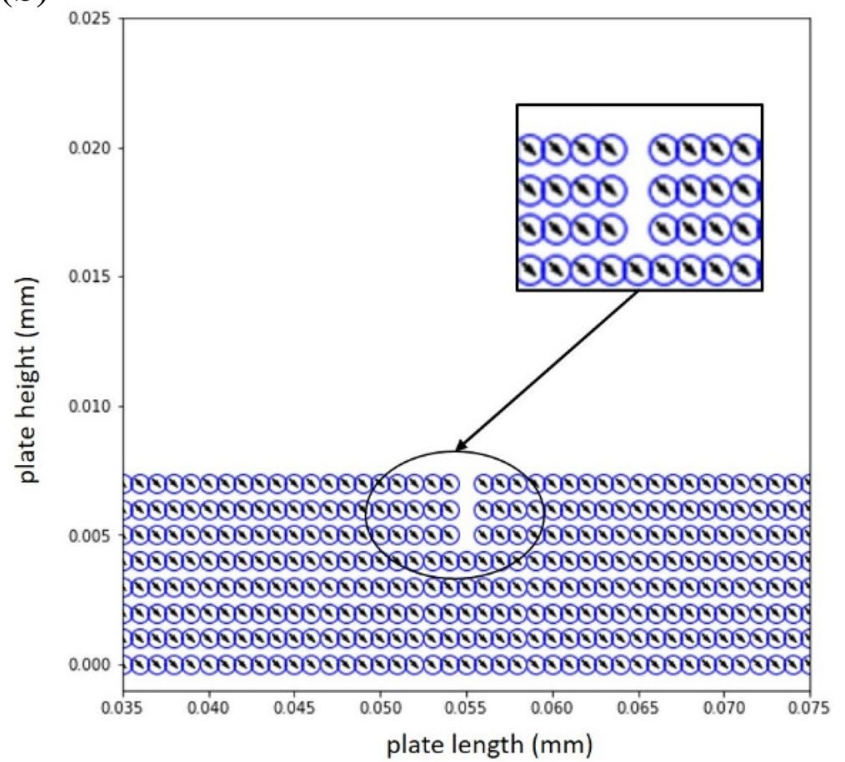

Fig. 7 a array of random magnetic domains, $\mathbf{b}$ ordered magnetic domains on the plate AST A-27 under test

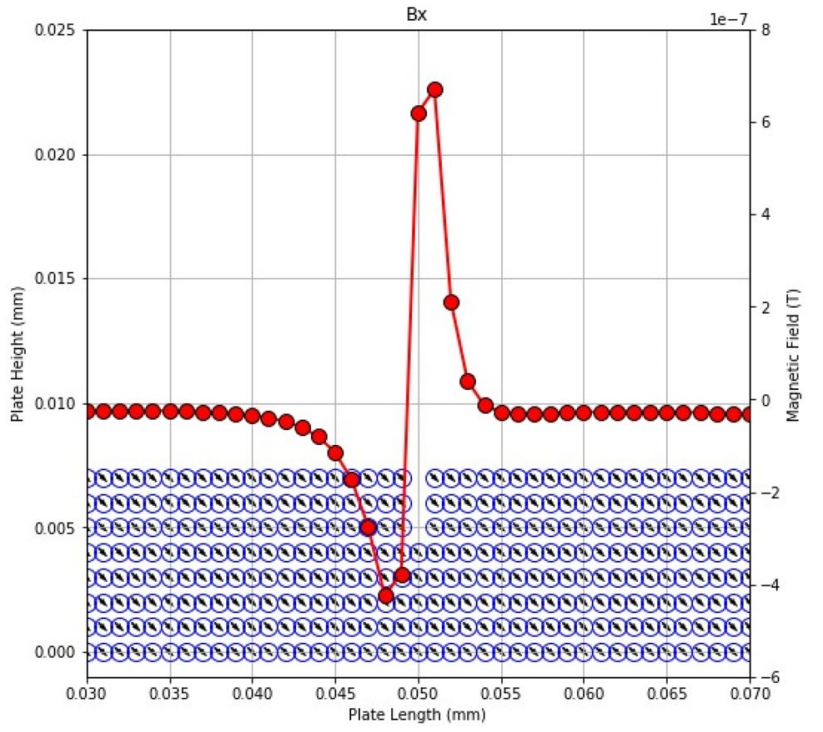

Fig. 8 Magnetic field $X$ of a rectangular defect with ordered magnetic domains

to the graphical user interface developed in Televiewer through the Bluetooth communication protocol. If the information is stored in the microSD, then it is processed on a PC.

The mobile robot uses a proportional integral derivative (PID) controller, which was designed to stabilize the speed using an LSM6DS33 3-axis accelerometer, the infrared sensors are used to handle the turning left, right and the

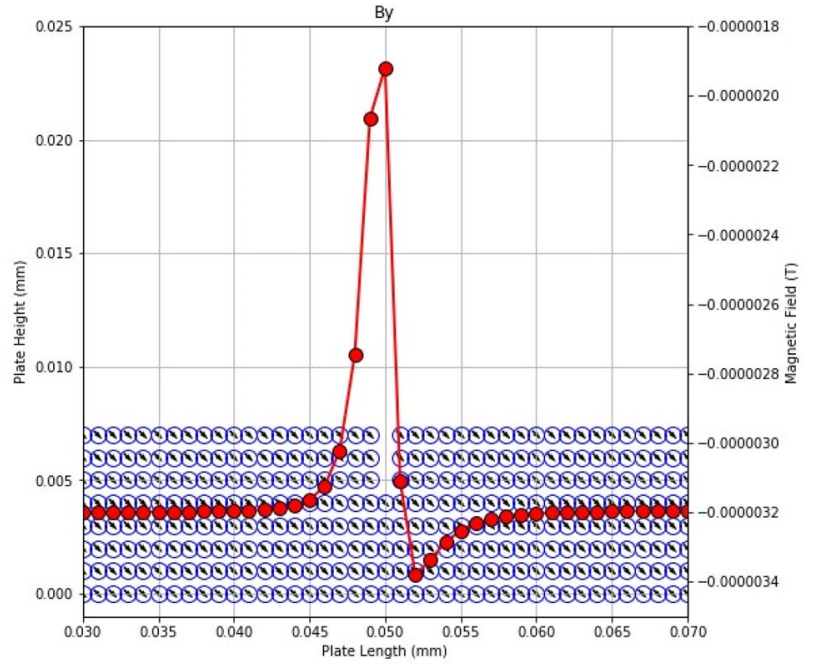

Fig. 9 Magnetic field $\mathrm{Y}$ of a rectangular defect with ordered magnetic domains

moving forward, backward and reverse. Figure 12 shows the structure of the control system for the mobile robot.

As can be observed in Figs. 13, 14 and 15, the maximum peak is $-1987 \mu \mathrm{T}, 3957 \mu \mathrm{T}$, and $3220 \mu \mathrm{T}$, respectively. This model is important, enabling that the experimental profiles are being almost reproduced. This type of comparisons allows to extract geometric characteristics related to the width and depth of the flaws.

Figures 16,17 and 18 , the maximum peak is $-1949 \mu \mathrm{T}$, $3613 \mu \mathrm{T}$, and $2681 \mu \mathrm{T}$, respectively. 


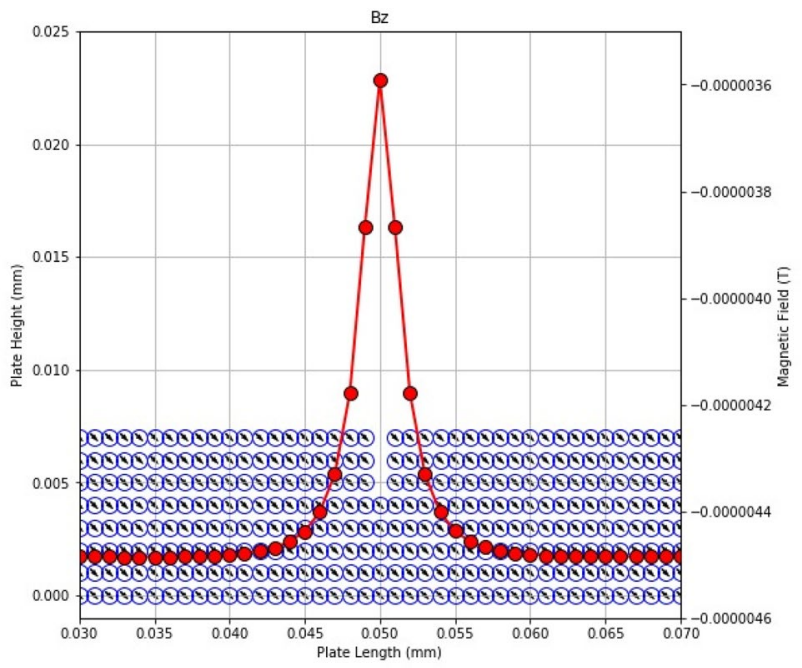

Fig. 10 Magnetic field $Z$ of a rectangular defect with ordered magnetic domains

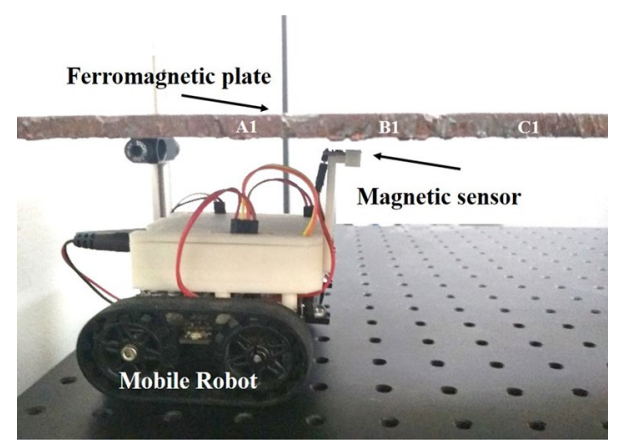

Fig. 11 Arrangement implemented for defect measurement on the ferromagnetic board

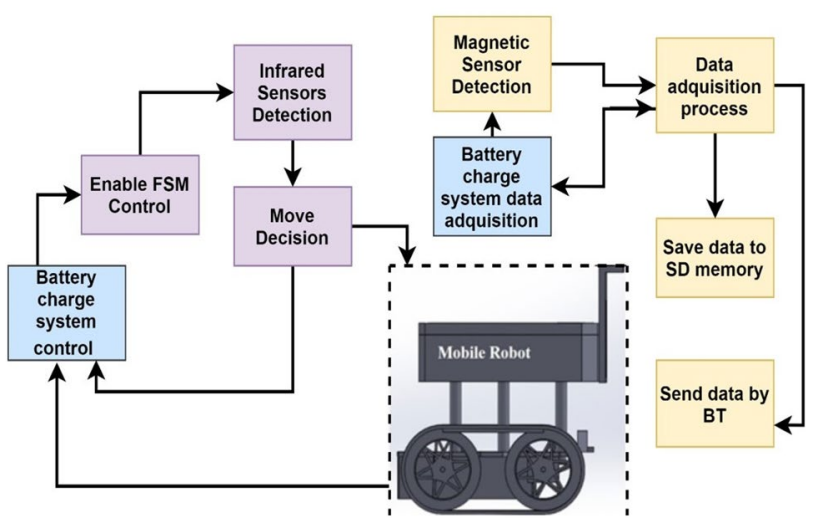

Fig. 12 Complete system for mobile robot control

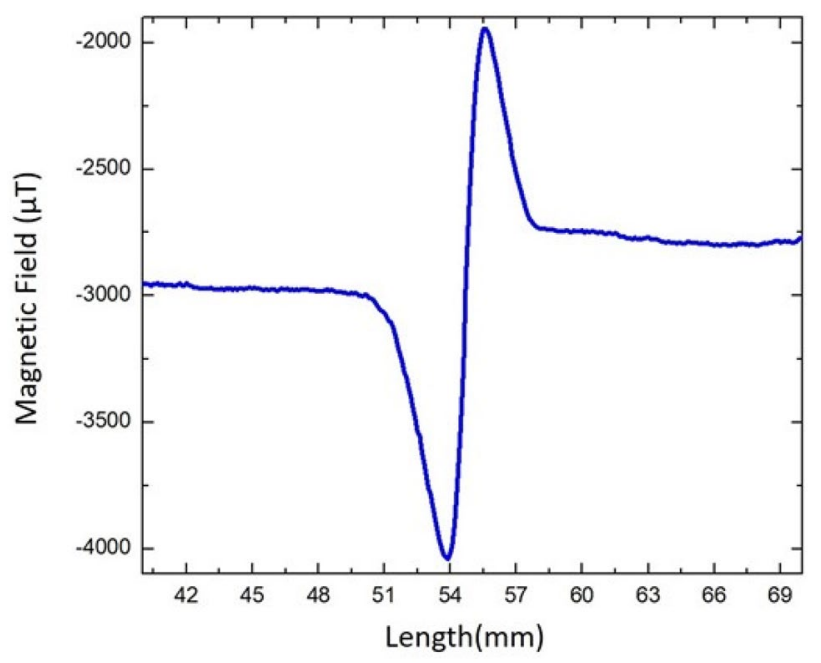

Fig. 13 Experimental result of magnetic field $X$ of a rectangular defect $A 1$ with ordered magnetic domains

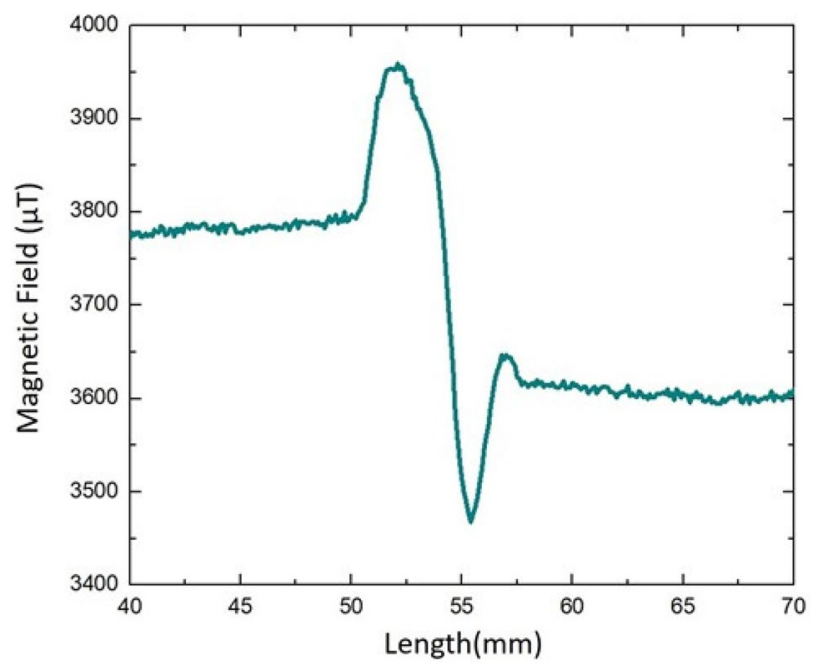

Fig. 14 Experimental result of magnetic field $Y$ of a rectangular defect $A 1$ with ordered magnetic domains

Finally, Fig. 19 shows the behavior obtained for flaw C1 where the maximum peak is at $-2559 \mu \mathrm{T}$ for the $\mathrm{X}$ axis, Fig. 20 shows for the $Y$ axis a maximum peak from -3304 $\mu \mathrm{T}$ in Fig. 21 shows the $Z$ axis the maximum peak is evident at $2724 \mu \mathrm{T}$.

\section{Discussion}

The mobile robot is capable to online defect monitoring on ferromagnetic structures. By using the MMM, no special treatment is required for sampling such as liquid penetrant techniques, emission of Eddy currents, industrial 


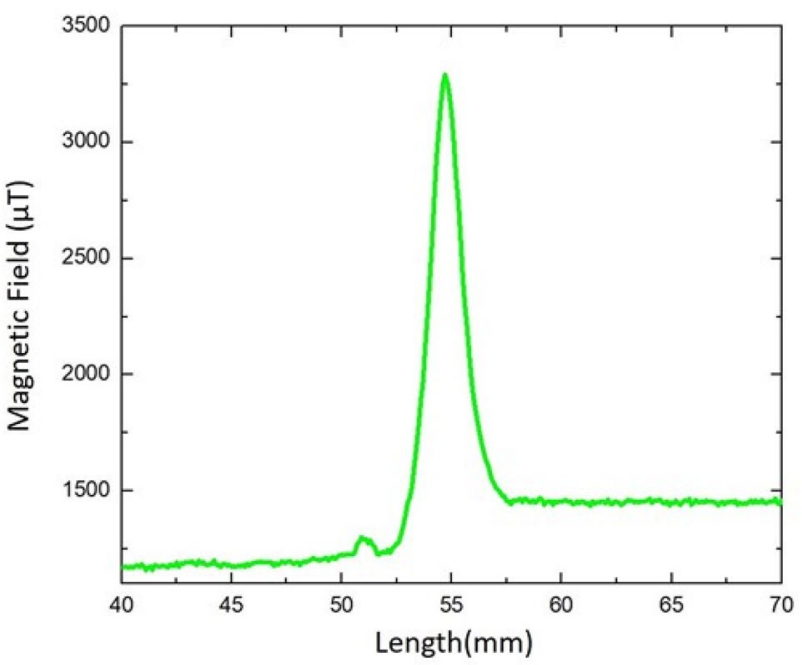

Fig. 15 Experimental result of magnetic field $Z$ of a rectangular defect $A 1$ with ordered magnetic domains

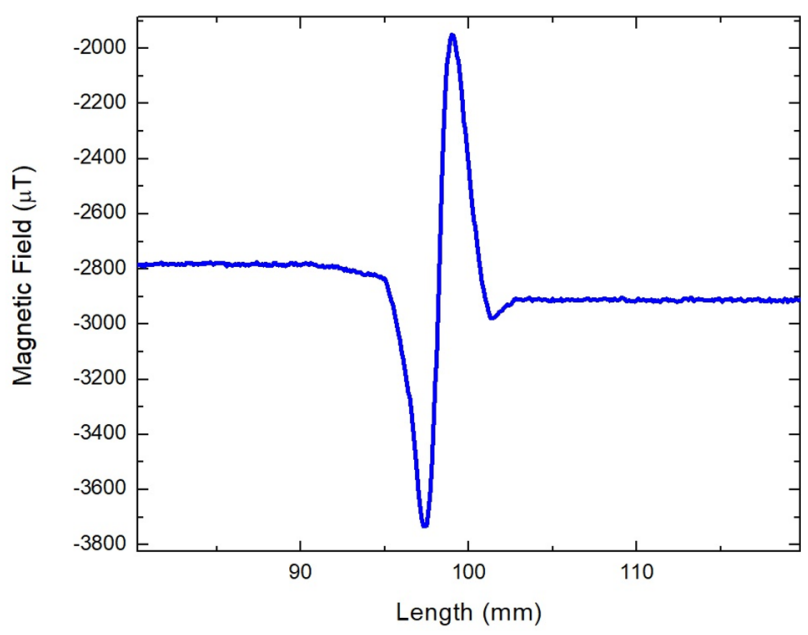

Fig. 16 Experimental result of magnetic field $X$ of a rectangular defect B1 with ordered magnetic domains

ultrasound, acoustic radiation or the use of destructive techniques. The proposed system reduces the use of expensive equipment and additional operators to monitor rectangular defects in ferromagnetic structures. The dynamic range for robot autonomy can be increased by using a higher value power supply or by modifying the path speed of the mobile robot.

\section{Conclusions}

In this paper a mobile robot was used to inspect rectangular defects in a ASTM A-27 ferromagnetic plate. The proposed prototype has two embedded systems, one for



Fig. 17 Experimental result of magnetic field $Y$ of a rectangular defect B1 with ordered magnetic domains



Fig. 18 Experimental result of magnetic field $Z$ of a rectangular defect $B 1$ with ordered magnetic domains

control and other for data acquisition, which is capable of measuring small variations of the magnetic field due to defects of the order of millimeters at a travel speed of $2.15 \mathrm{~cm} / \mathrm{s}$, so the prototype may be suitable for inspection tasks in preventive maintenance in ferromagnetic structures installed in industry settings to avoid serious accidents or environmental damage. The robotic prototype has an autonomy of $45 \mathrm{~min}$, while the microsensor has a resolution of $0.1 \mu \mathrm{T}$. The size and depth of the defects measured are related to the shape of the magnetic field measured, the variations present are due to deformations or cracks that are not visible.

The results of the work allow us to appreciate the possible practical applications of the MMM, the ability to analyze defects with a simple device such as the one 


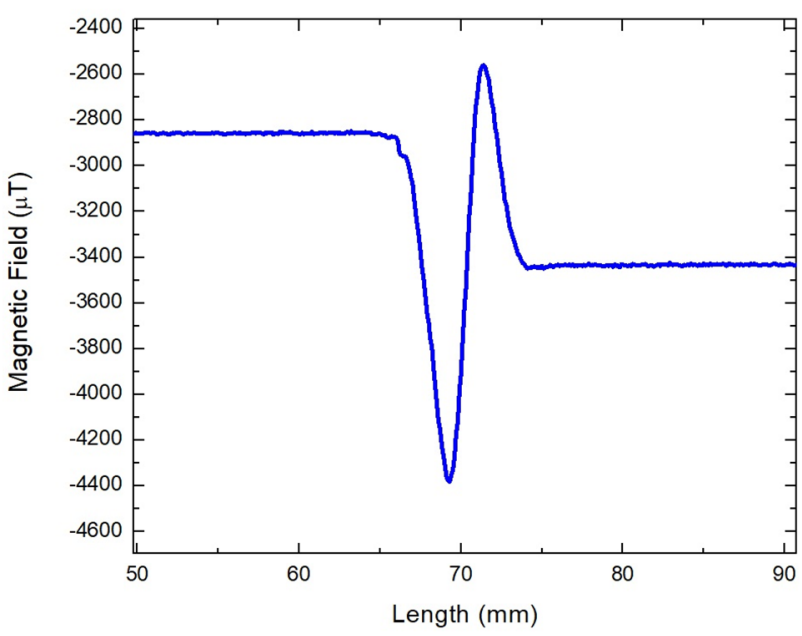

Fig. 19 Experimental result of magnetic field $X$ of a rectangular defect $\mathrm{C} 1$ with ordered magnetic domains

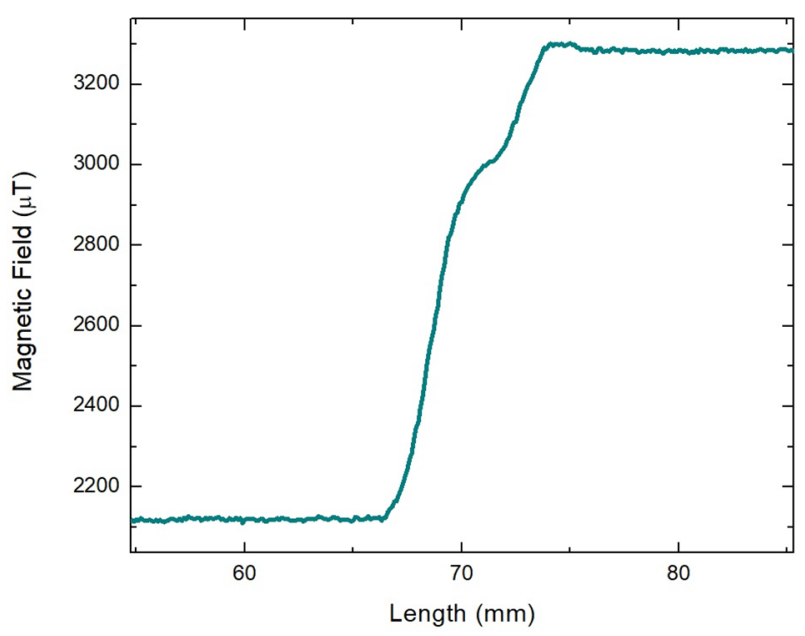

Fig. 20 Experimental result of magnetic field $Y$ of a rectangular defect $\mathrm{C} 1$ with ordered magnetic domains

proposed is an advantage to be taken into account with other inspection methods, at the same time, the possibility of adapting the device to the needs of the structures to be analyzed highlight its potential even more since the design of the device can be modified for its use not only in plates as shown in the results but also in pipes or steel beams. The graphic user interface is easily accessible and user-friendly, where the records obtained from the magnetoresistive microsensor can be monitored. The algorithm implemented in Python programming language is an important model to determine magnetic defects in non-destructive testing, the results shows a high correlation between the flaw simulated and experimental measurements.



Fig. 21 Experimental result of magnetic field $Z$ of a rectangular defect $\mathrm{C} 1$ with ordered magnetic domains

Future work will include the study of irregular defects according to the ASTM standard for intergranular or transgranular corrosion and the use of additional microsensors to determine the size and shape of the and FPGA for the embedded systems. Additionally, the universal algorithm implement in [23] for automatic control of movement of mobile wheeled robots using an inertial sensor data, can be integrated into the mobile robot presented in this work in order to record your position in the microSD memory. Specifically, it will be very useful to estimate the plate defects with the position where it occurs, during inspection processes.

Acknowledgements Nanoscience of the Research Center in Micro and Nanotechnology of University of Veracruz (MICRONA-UV), México, National Council of Science and Technology (CONACYT), trough scholarship 787729, and University of Antioquia for the exclusive dedication program.

Funding This research was funded by project PRODEP 511-6/198453 "Modelado y Desarrollo de Microsensores CMOS-MEMS para la detección de diabetes", Partial financial support was also provided by the CODI-UdeA Project No. 2017-16253. J.R

\section{Declarations}

Conflict of interest The author(s) declared no potential conflicts of interest with respect to the research, authorship, and/or publication of this article.

Open Access This article is licensed under a Creative Commons Attribution 4.0 International License, which permits use, sharing, adaptation, distribution and reproduction in any medium or format, as long as you give appropriate credit to the original author(s) and the source, provide a link to the Creative Commons licence, and indicate if changes were made. The images or other third party material in this article are included in the article's Creative Commons licence, unless indicated otherwise in a credit line to the material. If material is not 
included in the article's Creative Commons licence and your intended use is not permitted by statutory regulation or exceeds the permitted use, you will need to obtain permission directly from the copyright holder. To view a copy of this licence, visit http://creativecommons. org/licenses/by/4.0/.

\section{References}

1. Gholizadeh S (2016) A review of non-destructive testing methods of composite materials. Procedia Struct Integr 1:50-57

2. Villegas -Saucillo JJ, Díaz-Carmona JJ, Cerón-Álvarez CA, JuárezAguirre R, Domínguez-Nicolás SM, López-Huerta F, Herrera-May AL, (2019) Measurement System of Metal Magnetic Memory Method Signals around Rectangular Defects of a Ferromagnetic Pipe. Appl Sci 9:2695

3. Chen B, Liu J (2008) Damage in carbon fiber-reinforced concrete, monitored by both electrical resistance measurement and acoustic emission analysis. Constr Build Mater 22:2196-2201

4. Cerneck J, Bozek P, Pivarciova E (2015) A new system for measuring the deflection of the beam with the support of digital holographic interferometry. J Electr Eng 66:53-56

5. Sága M, Blatnická M, Blatnický M, Dižo J, Gerlici J (2020) Research of the fatigue life of welded joints of high strength steel 5960 QL created using laser and electron beams. Materials 13:2539

6. Yong-Kai Z, Gui-Yun T, Rong-Sheng L, Hong Z (2011) A review of optical NDT technologies. Sensors 11:7773-7798

7. Doubov AA (2012) Development of a metal magnetic memory method. Chem Pet Eng 47:837-839

8. Bulychev OA, Shleenkov AS (2008) A Magnetic Scanner Based on Residual Magnetization Used for Testing Ferromagnetic Articles. Russ J Nondestruct test 44:113-116

9. Wongi NS (2017) Distinguishing crack damage from debonding damage of glass fiber reinforced polymer plate using a piezoelectric transducer based nondestructive testing method. Compos Struct 159:517-527

10. Chen N, Lin H, Xiaokai W (2018) Crack propagation analysis and fatigue life prediction for structural alloy steel based on metal magnetic memory testing. J Magn Magn Mater 462:144-152

11. Erlong L, Yihua K, Jian T, Jianbo W (2018) A New Micro Magnetic Bridge Probe in Magnetic Flux Leakage for Detecting Microcracks. J Nondestruct Eval 37:36-47
12. EickhoffW (1981) Temperature sensing by mode-mode interference in birefringent optical fibers. Opt lett 6:204-206

13. Beltrán-Pérez G, Kuzin EA, Carnas-Anzuelo J, López R, Spirin VV, Marquez-Lucero A (2002) Fiber bend losses produced by soft and swellable materials for hydrocarbon detection. Optics Comm 204:145-150

14. Anvo R, Sattar TP, Gan TH, Pinson I (2018) Non-destructive testing robots (NDTBOTs) for In-service storage tank inspection. J Mech Eng Auto 8:103-109

15. Mineo C, Herbert D, Morozov M, Pierce SG 2012 "Robotic NonDestructive Inspection", In: 51st Annual Conference of the British Institute of Non-destructive Testing, Daventry, Northhamptonshire, UK, 345-352.

16. Emmanouilidis C, Spais V, Hrissagis K 2004 A mobile robot for automated non-destructive testing of steel plates, Proc of the IEEE Mechatronics and Robotics

17. Silva MF, Barbosa RS, Oliveira ALC (2012) Climbing Robot for Ferromagnetic Surfaces with Dynamic Adjustment of the Adhesion System. J Robotics 2012:1-16

18. Isawa K, Nakayama S, Ikeda M, Takagi S, Tosaka S, Kasai N (2005) Robotic 3D SQUID imaging system for practical nondestructive evaluation applications. Physica C 432:182-192

19. Seung-Nam Y, Jae-Ho J, Chang-Soo H (2007) Auto inspection system using a mobile robot for detecting concrete cracks in a tunnel. Autom Constr 16:255-261

20. Kilin A, Bozek P, Karavaev Y, Klekovkin A, Shestakov V (2017) Experimental investigations of a highly maneuverable mobile omniwheel robot. Int J Adv Robotic Syst 14:1-9

21. López-Huerta F, Rojas-Nava G, Soto-Cruz BS, Herrera-May AL (2013) Diseño e implementación de un sistema de caracterización para microsensores de efecto Hall. Rev Mex Fis 59:54-61

22. Freescale Semiconductor. Available online:https://www.nxp. com/docs/en/data-sheet/MAG3110.pdf (accessed on 12 October 2019)

23. Integration of inertial sensor data into control of the mobile platform, Springer International Publishing, Advances in Intelligent Systems and Computing 511: 271-282 (2017)

Publisher's Note Springer Nature remains neutral with regard to jurisdictional claims in published maps and institutional affiliations. 